\section{Intracellular Speciation and Nanoscale Distribution of Silver and Bioessential Metals in Fish Intestinal Epithelia upon Exposure to Nanoparticles}

\author{
JEFFREY G CATALANO ${ }^{1}$, WILLIAM DUDEFOI ${ }^{1,2,3}$,
} ELAINE FLYNN $^{1}$ AND MATTEO MINGHETTI ${ }^{2}$

\author{
${ }^{1}$ Washington University in St. Louis \\ ${ }^{2}$ Oklahoma State University \\ ${ }^{3}$ Eawag, Swiss Federal Institute of Aquatic Science and \\ Technology \\ Presenting Author: catalano@wustl.edu
}

Metal nanoparticles are widely released into aquatic systems and taken up by diverse organisms. Such exposure may cause toxicity effects, both directly and from modification of intracellular processes. Here we investigate the impact of exposure to $\mathrm{Ag}$ and $\mathrm{TiO}_{2}$ nanoparticles on intestinal cells of rainbow trout. These studies utilize a novel cell culture, RTgutGC, derived from the intestinal fish epithelium that display polarization and tight junction when grown on porous membranes. This enables studies of nanoparticle exposure linked to cellular function and toxicity. We have sought to connect these observations to the intracellular speciation and distribution of bioessential metals, which are normally regulated via homeostasis, using X-ray spectroscopic and imaging methods. All data were collected under cryogenic conditions to prevent beam-induced cellular damage and alter metal speciation.

X-ray absorption spectroscopy reveals that, following uptake, Ag nanoparticles partially alter via intracellular Ag-thiol complexation, which correlates with an increase in metallothionein mRNA levels. Exposure to dissolved Ag produces almost exclusively Ag-thiol complexes and is associated greater toxicity compared to a similar Ag nanoparticle exposure. Intracellular $\mathrm{Zn}$ and $\mathrm{Cu}$ speciation are unaffected by exposure to dissolved $\mathrm{Ag}$ as well as to both $\mathrm{Ag}$ and $\mathrm{TiO}_{2}$ nanoparticle. Ag was excreted from cells following exposure to both dissolved and nanoparticle forms of Ag. Nanoscale X-ray fluorescence imaging showed that $\mathrm{Ag}$ and $\mathrm{TiO}_{2}$ nanoparticles form 0.4 to $3 \mu \mathrm{m}$ intracellular clusters, including near the nucleus. Minor $\mathrm{Ag}$ is also detectable in the cytoplasm and nucleus in areas free of particles, indicating partial dissolution, but $\mathrm{TiO}_{2}$ is only detectable in particulate form. While the nanoparticle studies were conducted at non-toxic exposure levels, introduction of toxic concentrations of dissolved $\mathrm{Ag}$ resulted in concentration of this element in the nucleus and induced nuclear shrinkage, possibly associated with a preapoptotic mechanism. This work provides new insight into the intracellular transformations of metal nanoparticles in the environment by aquatic organisms. Intracellular dissolution, potentially aided by thiol complexation, followed by excretion from intestinal epithelia introduces $\mathrm{Ag}$ derived from nanoparticles into the bloodstream. These internal transformations of $\mathrm{Ag}$ nanoparticles likely result in eventual 